\title{
Application of VRP Model in the Transport of Water Resources in Henan Province
}

\author{
Ziqiao Wang ${ }^{1, a}$ \\ ${ }^{1}$ School of North China Electric Power University, Baoding 071003, China; \\ âwangziqiao722@sina.com
}

Keywords: Water Resources, Transportation, VRP, GA

\begin{abstract}
Considering the shortage of water resources in Henan Province, we draw up an intervention program to mitigate water scarcity. The model described in this article is established for researches of transporting water from the Danjiangkou reservoir to Henan Province. The water movement reduces the uneven distribution of water resources, and increases the amount of available water, while it may destroy the balance of the water cycle. We build the VRP (Vehicle Routing Problem) model to solve the problem, and use the GA (Genetic Algorithm) to calculate the order and the ratio of water movement.
\end{abstract}

\section{Analysis}

According to the causes of the shortage of water resources in Henan Province, the physical driver of water scarcity in Henan is the amount of available water, and the economical driver is the storage and pollution of water. The plan of water movement can benefit both the physical and economical scarcity. So we determine the VRP (Vehicle Routing Problem) model to solve the problem.

\section{The Establishment of Model}

We choose a place full of water as the water source to supply water to the cities in Henan. We assume the demand water of city $i$ is $g_{i}$, the facility of water movement from the source can carry water $q_{n}$, which passes $n$ cities, and finally go back to the source. Our goal is to arrange the water movement routes reasonably to cover the demand of all cities and make sure the routes is shortest.

The constraint conditions are as follows:

1. The traffic of facility can't be more than the maximum of its water carrying capacity.

2. Each city has only one facility across.

3. The distance of each route can't exceed the maximum distance the facility can reach.

4. The limitation of water demand time must be considered.

The goal of the VRP model is to use the least cost to cover all cities' water demand. In general, the cost of water traffic is proportional to the distance of route. The shorter routes are, the less cost is. So we build the VRP model with the goal of least routes distance as below.

$$
\begin{gathered}
\min D=\sum_{i=0}^{h} \sum_{j=0}^{h} \sum_{s=1}^{k} c_{i j} x_{i j s} \\
\sum_{i=0}^{h} x_{i j s}=y_{i s}, j=1,2, \cdots, h, \forall s \\
\sum_{j=0}^{h} x_{i j s}=y_{i s}, i=1,2, \cdots, h, \forall s
\end{gathered}
$$




$$
\begin{gathered}
\sum_{i=0}^{h} g_{i} y_{i s} \leq q_{s}, \forall s \\
\sum_{s=0}^{k} y_{i s}= \begin{cases}1 & i=1,2, \cdots, h \\
k & i=0\end{cases}
\end{gathered}
$$

Where $c_{i j}$ stands for the cost of traffic from city $i$ to city $j . x_{i j s}$ standls for the traffic, when facility $s$ travel from $i$ to $j, x_{i j s}$ is equal to 1 , otherwise it is equal to $0 . y_{i s}$ shows the task of city $i$ water supply is accomplished by facility $s$, if it happens, it is 1 . If not, it is $0 . q_{s}$ represents the maximum of water carrying capacity of the facility $s$.

Because VRP is the expansion of TSP (Traveling Salesman Problem), we use the method of solving the TSP problem, which is GA (Genetic Algorithm). We can code each city by natural number. As the code of traffic facilities, we insert $m-1$ zeros to divide the sequence into $m$ segments, each of them represents a route of water movement. Then we can transfer the problem of VRP to TSP, and use GA to deal with it.

\section{The Evaluation of Model}

In fact, this model may destroy the balance of water cycle, and have a negative effect on the ecological environment. But this model also can improve the water supply in some areas, and it can reduce the uneven distribution of water resources. As for Henan, the amount of water changes little, it is suitable to adopt the water movement plan.

\section{The Results and Conclusions}

Aiming at the water movement in Henan, we decide to select the Danjiangkou reservoir as the water source of the movement. The Danjiangkou reservoir covers an area of 1022.75 Square kilometers, and the storage amount reaches 290.5 billion $\mathrm{m}^{3}$. Its location is as the below.

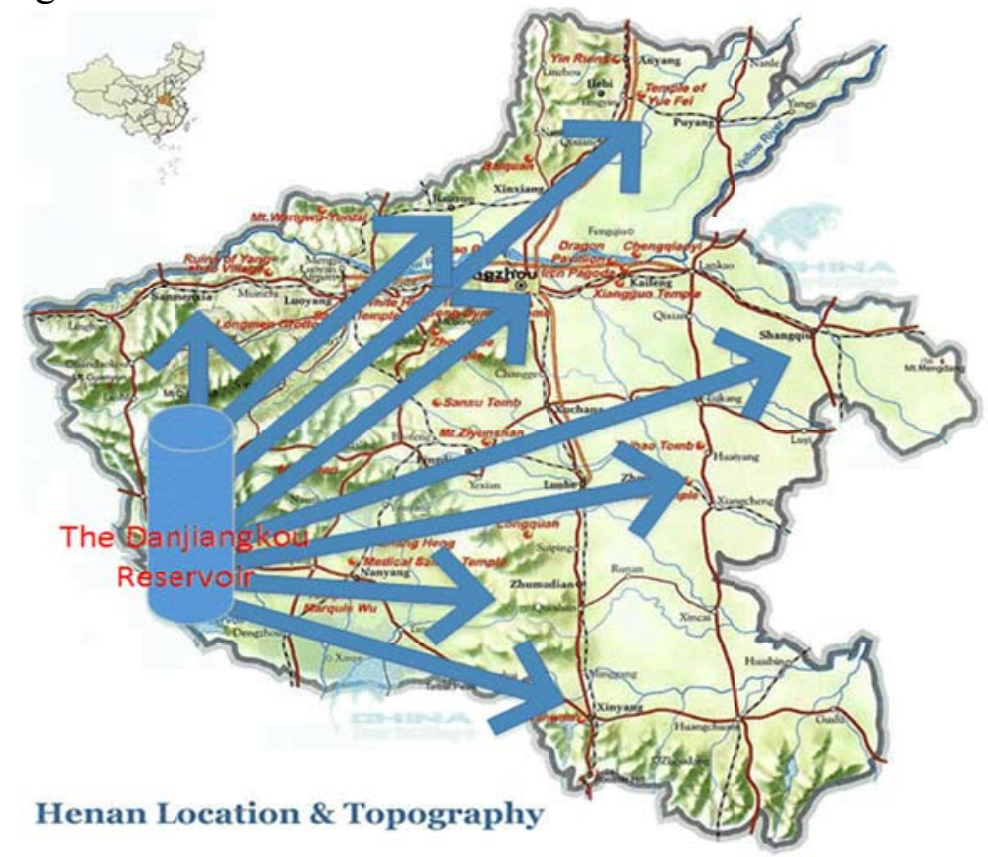

Fig.1 The map of Danjiangkou and Henan

According to the reference [1], we can know the prediction of water resources in Henan Province in future.Since the forecast of water demand and supply of Henan in 2016 2030 is known, we can choose 2016 for example. In 2016, Henan lacks 33.54 billion $\mathrm{m}^{3}$ to meet the demand. Based on the historical data, we get the ratios of water that should be carried to each city. Based on the ratios, we determine how much water should be transported from Danjiangkou reservoir to cities in Henan. 
Substitute the data to the VRP model, after 100 iterations in GA, we obtain the order and the ratios as below.

Tab.1 The order and the ratios of water movement

\begin{tabular}{cccc|cccc}
\hline Number & City & Ratio & Order & Number & City & Ratio & Order \\
\hline 1 & Luohe & 0.58 & 11 & 10 & Xuchang & 1.09 & 10 \\
2 & Luoyang & 2.01 & 3 & 11 & Jiyuan & 0.34 & 18 \\
3 & Anyang & 2.03 & 5 & 12 & Zhumadian & 1.51 & 17 \\
4 & Zhoukou & 2.83 & 16 & 13 & Nanyang & 3.62 & 13 \\
5 & Xinyang & 2.38 & 15 & 14 & Sanmenxia & 0.63 & 12 \\
6 & Puyang & 2.25 & 9 & 15 & Jiaozuo & 2.01 & 8 \\
7 & Xinxiang & 2.57 & 7 & 16 & Zhengzhou & 2.50 & 1 \\
8 & Hebi & 0.74 & 6 & 17 & Kaifeng & 2.81 & 2 \\
9 & Pingdingshan & 1.44 & 4 & 18 & Shangqiu & 2.20 & 14 \\
\hline
\end{tabular}

\section{References}

[1] Ji-chang Z. The Balance Analysis and Forecast on Supply and Demand of Water Resources in Henan Based on Grey Model[J]. Journal of Zhongyuan University of Technology, 2014, 3: 014.

[2] Stone C J. Consistent nonparametric regression[J]. The annals of statistics, 1977: 595-620.

[3] Colby B G. Enhancing instream flow benefits in an era of water marketing[J]. Water Resources Research, 1990, 26(6): 1113-1120.

[4] Xiao-bin Y. Analysis about the Rainfall Condition During the Recent Ten Wet Seasons in Pudong New Area[J]. Water Sciences and Engineering Technology, 2012, 2: 005.

[5] Xiang-yang L I. Genetic algorithm for VRP[J]. Computer Engineering and Design, 2004, 31(5): 271-276.

[6] Yan-fang N. Study on the Model and Algorithm for Vehicle Routing Problem [J][J]. Shanxi Electronic Technology, 2010, 1: 026.]

[7] http://www.city8.com/map/1518.html 